\title{
Therapeutic Interventions Using Ursolic Acid for Cancer Treatment
}

\section{Ray Navin and Soo Mi Kim*}

Department of Physiology, Institute for Medical Sciences, Chonbuk National University Medical School, Jeonju, Republic of Korea

\begin{abstract}
Current research has supported the potential of plant-derived natural compounds for the treatment and prevention of cancer and ursolic acid (UA) is one such compound. Unraveling the molecular mechanism of the protective effects of UA could establish a novel foundation to treat cancer. Studies have demonstrated that UA targets intracellular and extracellular molecules that are responsible for processes such as apoptosis, metastasis, autophagy, angiogenesis, oxidative stress, and inflammation. UA can reportedly be used as a potential drug when integrated with modern technological advancements to treat cancer and other diseases. In this review, the availability, chemical structures and properties, bioactivity, and the different molecular mechanisms of UA against cancer are compiled to understand the multiple aspects as well as the limitations of this potential treatment.
\end{abstract}

Keywords: Ursolic acid; Cancer; Metastasis; Autophagy; Angiogenesis; Oxidative stress; Inflammation

\section{Introduction}

From an ancient time, plants and herbs have been used as a healing agent in a variety of ailments, and almost every plant, vegetable, and fruit contains different bioactive compounds (produced through secondary metabolism) that have numerous therapeutic uses [1]. Among the different groups of these bioactive phytochemicals, triterpenoid is one of the important compounds used as an anti-inflammatory, anticancer, and anti-microbial agent [2]. Triterpenoids are universally found in plants; specifically, pentacyclic triterpenes have distinctive biological properties as shown by emerging research [3]. American Cancer Society (2015) declared cancer as the second leading cause of death in the US, with expectations to overtake heart disease in the next few years [4]. Similarly, the World Health Organization (WHO) estimates that cancer causes the most fatalities compared with any other disease worldwide [5]. However, modern advances in the field of medical sciences have decreased the number of cancer deaths over the last two decades $[4,5]$. In addition to surgery and radiotherapy, natural compounds, particularly triterpenoids such as ursolic acid (UA), have shown great potential to inhibit cancer development [6]

UA is a $3 \beta$-hydroxy-12-urs-12ene-28-oic acid and is one of the pentacyclic triterpenoids present in different plants, herbs, and fruits (e.g., apples, pears, and figs) [7]. Extensive research has been conducted over the last few decades to clarify the role of UA in various diseases, especially cancer [8,9] (Figures 1 and 2). Some of the plants that contain UA include Malus domestica, Origanum majorana, Rosmarinus officinalis, Salvia officinalis, Thymus vulgaris, Eucalyptus, and Coffea arabica, in addition to the outer peel of many different fruits [10]. Ongoing studies have demonstrated that UA is capable of inducing apoptosis, arresting cell cycle progression, inhibiting cellular proliferation, and reducing tumorigenesis in some cancer cells via multiple signaling pathways $[2,11]$. Bonaccorsi et al. found that UA suppresses endogenous reverse transcriptase (RT), an enzyme which regulates cellular proliferation and differentiation, in both melanoma (A375) and anaplastic carcinoma (ARO) cells [12]. Because UA is not soluble in water, its bioavailability is lower in the body, making it difficult to acquire its full advantage [13]. Potential anti-cancer anti-cancer effects of UA include anti-proliferative, proapoptotic, anti-metastatic, and anti-angiogenic effects [14], and pharmacological properties of UA include anti-inflammatory, anti-oxidative stress, anti-mutagenic, antiatherosclerotic, anti-hypertensive, anti-leukemic, anti-viral, and antidiabetic effects [15]. In addition, a recent phase I clinical trial conducted by Qian et al. found that UA efficiently enhances the patient remission rate with an acceptable level of toxicity in 21 subjects with solid tumors
[16]. Therefore, the goal of this review is to summarize the effects of UA on various types of cancer and discuss the signaling, mechanisms, and pathways involved in the prevention and treatment of cancer.

\section{Chemistry of UA}

The structure of UA comprises C-30 isoprenoid in a pentacyclic triterpenoid, and UA has low solubility in water but is highly soluble in glacial acetic acid and alcoholic $\mathrm{NaOH}$. Its physical appearance is a crystalline solid, with fine hair-like spikes, with a melting point of $284^{\circ} \mathrm{C}$ and a molecular weight of $456.70032 \mathrm{~g} / \mathrm{mol}$ [14].

\section{Therapeutic Roles}

\section{Apoptosis and cell cycle arrest}

Apoptosis, or programmed cell death, is specific morphological and biochemical alterations leading to natural cell death. Apoptosis has a very significant role in controlling various biological processes, such as embryonic development, hematopoiesis, and immunity [17]. Kim et al. showed that UA induces apoptosis via activating caspase-3 in a HepG2 cell line [18]. The features of apoptotic cells include

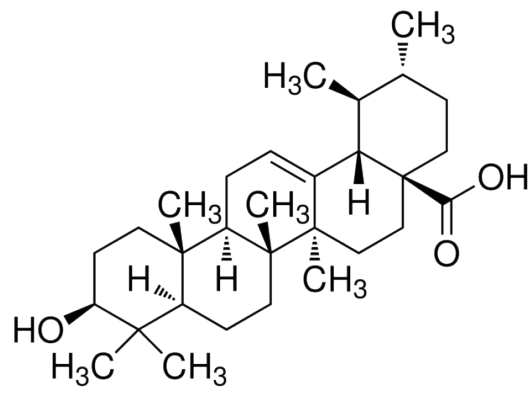

Figure 1: Chemical structure of UA.

*Corresponding authors: Soo Mi Kim, Department of Physiology, Institute for Medical Sciences, Chonbuk National University Medical School, Gungiro 20 , Deokjin-Gu, Jeonju 561-180, Republic of Korea, Tel: +82-63-270-3077; E-mail: soomikim@jbnu.ac.kr

Received May 09, 2016; Accepted May 19, 2016; Published May 24, 2016

Citation: Navin R, Kim SM (2016) Therapeutic Interventions Using Ursolic Acid for Cancer Treatment. Med chem (Los Angeles) 6: 339-344. doi:10.4172/21610444.1000367

Copyright: (C) 2016 Navin R, et al. This is an open-access article distributed under the terms of the Creative Commons Attribution License, which permits unrestricted use, distribution, and reproduction in any medium, provided the original author and source are credited. 
DNA fragmentation, cellular shrinkage, an increase in cytoplasmic $\mathrm{Ca}^{2+}$ levels, cytoplasmic and nuclear membrane blebbing, as shown in HL-60 (human promyelocytic leukemia) cells and Daudi (human B-lymphoblastoid) cells after treatment of UA $[8,19]$. UA reportedly suppressed epidermal growth factor receptor/mitogen-activated protein kinase (EGFR/MAPK) pathway to induce apoptosis and inhibit cellular proliferation [20]. Moreover, Weng et al. found that UA significantly decreased the proliferation of gall bladder cancer cells (i.e., GBC-SD and SGC-996 cells), using an MTT assay in a dosedependent manner, and that US caused an S-phase cell cycle arrest in both cell types and increases the expression of pro-apoptotic markers (i.e., activated caspases and PARP), using an immunoblot assay. The authors also performed an in vivo study using a xenograft model and observed tumor growth inhibition zin nude mice [21]. Moreover, an in vitro study of bladder cancer demonstrated that UA induces ER stress in T24 cells, which in turn activates a Jun N-terminal kinase (JNK) signaling pathway to trigger eventual apoptosis [22]. A study involving breast cancer cells (i.e., MCF-7 and MDA-MB-231) revealed that the induction of apoptosis occurred via the mitochondrial death pathway, the extrinsic death receptor pathway, and by downregulating the expression of FoXM1 protein. Wang et al. also found that in MCF-7 cancer cells, UA initiates apoptosis, as well as downregulates cyclinD1/ CDK4, resulting cell cycle arrest [23,24]. In addition, a study of cervical cancer (i.e., HeLa and SiHa) cells found that UA has the potential to induce apoptosis via a mitochondrial intrinsic pathway and the inhibition of the ERK1/2 MAPK pathway [25]. Treatment of colorectal cancer with UA targets the phosphatidyl inositol 3 kinase (PI3K) signaling pathway to induce apoptosis in HCT15 and CO115 cells [26]. Moreover, the upregulation of the expression of p53, NF- $\kappa \mathrm{B}$, and Bax followed by enhanced transcriptional p21 activity and the activation of caspase- 3 and caspase- 9 was shown to be regulated by UA in human colon adenoma (SW480) cells [27]. Another group of researchers has reported the efficient induction of apoptosis in gastric cancer (SNU484, BGC-803) cells via the downregulation of Bcl-2, the upregulation of proteolytic activation of caspase- 3 and caspase- 8 , and the arrest of the cell cycle at the G0/G1 stage [9,28]. Glioma (U251) cells also exhibit an inhibition of proliferation and the initiation of apoptosis via the activation of caspase-3 in a dose-dependent manner [29]. The cell cycle arrest in the sub-G1 phase of human hepatocellular carcinoma (HUH7) cells is accompanied by apoptosis involving the discharge of cytochrome $\mathrm{c}$ into the cytosol, the activation of caspase- 3 and caspase- 9 , as well as the consequential cleavage of poly (ADP) ribose polymerase (PARP) [30]. This cleavage includes the downregulation of mRNA expression of the X-linked inhibitor of apoptotic protein (XIAP) in a dose-dependent manner following the treatment of UA [30]. In studies of melanoma, Harmand et al. demonstrated that UA is responsible for the induction of apoptosis in M4Beu cells via the mitochondrial intrinsic pathway and caspase- 3 activation, which is associated with an increase in Bax expression and a decrease in Bcl-2 levels after treatment [31]. Furthermore, Manu et al. found that UA induces apoptosis in B16F-10 cells, verified by the observation of apoptotic bodies and DNA fragmentation through the upregulation of p53 and caspase-3 [32]. This change was also associated with the downregulation of the anti-apoptotic gene Bcl-2 and melanoma progression in vitro [32]. Furthermore, Wu et al. investigated leukemia (K562) cells and showed that UA is able to inhibit cellular growth by inducing apoptosis through the upregulation of PTEN gene expression and inhibiting the PI3K/ Akt/mTOR pathway [33]. A study by Leal et al. demonstrated that UA initiates cell cycle arrest and apoptosis in pancreatic cancer (AsPC-1) cells with an upregulation in the levels of p53, p21 (waf1), and Noxa proteins [34]. One study on HCT15 (human colon cancer) cells revealed that UA resulted in a cell cycle arrest of the G0/G1 phase [35]. Combined with the initiation of apoptosis, UA is also responsible for the cell cycle arrest via cyclin and CDK (cyclin dependent kinase). Therefore, these accumulated results suggest that UA causes apoptosis in cancer cells by activating cell cycle arrest and altering related molecular targets that promote its anti-cancer properties.

\section{Anti-metastasis}

Metastasis causes the spread of cancer from one organ to another in an unpredictable manner. This renders effective treatments (e.g., surgery and radiotherapy) futile and leaves chemotherapy as the only other alternative; hence, several chemicals and natural compounds have been shown to inhibit metastasis of cancer [36,37]. One study showed that UA and its derivatives inhibit the metastatic effect of HepG2 cancer cells by suppressing the focal adhesion signaling pathway along with the regulation of cancer biomarkers such as ICAM-1, VCAM-1, E-selectin, FAK, paxillin, and PTEN. Additionally, immunohistochemistry revealed that UA also regulates the metastatic biomarker ICAM1 [37]. Tumor invasion linked to proteases, such as urokinase and cathepsin B, is also suppressed by UA [38]. A study of C6 glioma cells found that UA inhibits the expression of matrix metalloproteases

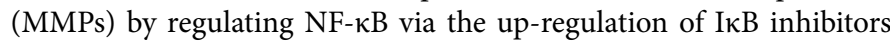
[39]. UA also inactivates MAPK/P38 signaling responsible for the augmentation of MMP expression in SNU484 cells [9]. Moreover, Liu et al. found that UA inhibits lung cancer metastasis in a dose-dependent manner in vitro. They found increased expression in E-cadherin as well as decreased expression in $\mathrm{N}$-cadherin and vimentin, which are responsible for the epithelial-mesenchymal transition (EMT). Additionally, UA inhibits metastasis by decreasing the expression of astrocyte elevated gene-1 (AEG-1) and inhibiting NF- $\mathrm{kB}$ [40]. In another study, Prasad et al. revealed that UA considerably decreases tumor volume in vivo and downregulates metastatic protein expression (e.g., MMP-9, VEGF, and ICAM-1) in vitro [41]. Moreover, Yeh et al. observed that UA causes a reduction in the migration and invasion of breast cancer (MDA-MB-231) in a dose- and time-dependent manner. This effect was associated with the diminished activities of MMP-2 and $\mathrm{u}$-PA by the inhibition of phosphorylation of Jun N-terminal kinase, Akt, and a mammalian target of rapamycin [42]. An in vitro analysis of highly metastatic human fibrosarcoma (HT1080) cells showed that UA triggers the downregulation of MMP-9 to reduce the extent of the tumor cell invasion via an invasion assay in the membrane of a transwell chamber [43]. Similarly, Huang et al. found that UA inhibits metastasis through the downregulation of MMP-9 in a dose-dependent manner, suppresses IL- $1 \beta$, subdues TNF- $\alpha$-induced activation of protein kinase C- $\zeta$ (PKC- $\zeta$ ), and suppresses the association of ZIP/p62 with PKC- $\zeta$ [39]. Moreover, to investigate the anti-metastatic property of UA, a mouse model of prostate cancer was treated with UA, and it was found that UA inhibits metastasis in vivo [44]. In addition, it was observed that UA downregulates the expression of CXCR4, subsequently reducing the expression of CXCL12-induced metastasis in prostate cancer cells in vitro [44]. Therefore, these obtained evidence elicits the potential of UA to suppress cancer by hindering metastasis via different signaling pathways.

\section{Anti-angiogenesis}

Angiogenesis is the development of new blood capillaries from previous blood vessels, for example, for tumor growth and its capacity for invasion. A study on Ehrlich ascites carcinoma (EAC) tumors by Saraswati et al. revealed that UA reduced peritoneal angiogenesis in EAC tumors compared to the controls in an in vivo model of angiogenesis [45]. Moreover, immunostaining results demonstrated a decrease in PECAM/CD31 (an endothelial cell marker) in UA-treated tumor sections, whereas the control tumor sections exhibited pronounced 
Citation: Navin R, Kim SM (2016) Therapeutic Interventions Using Ursolic Acid for Cancer Treatment. Med chem (Los Angeles) 6: $339-344$. doi:10.4172/2161-0444.1000367

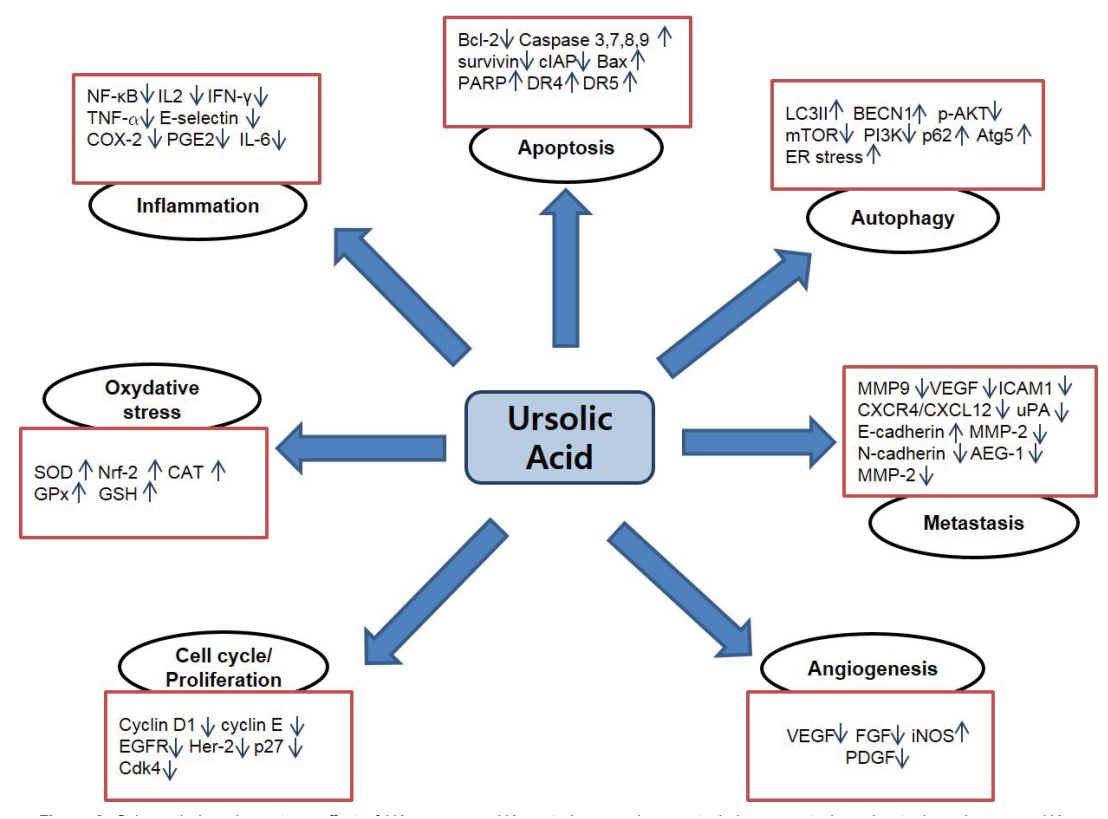

Figure 2: Schematic broad spectrum effect of UA on cancer. UA controls several genes to induce apoptosis and autophagy in cancer. UA regulates several genes to attenuate metastasis, angiogenesis, cell cycle/proliferation, oxidative stress, and inflammation in cancer. Lined up arrow indicates the increased gene levels whereas lined down arrow indicates the decreased gene levels after treatment of UA in cancer.

vascularity [45]. Lin et al. reported that UA treatment inactivates signal transducer and activator of transcription-3 (STAT3), as well as the AKT and sonichedgehog $(\mathrm{SHH})$ pathways responsible for tumor proliferation, invasion, and angiogenesis [11]. Lin et al. used UA to treat human liver cancer (i.e., Hep3B, Huh7, and HA22T cells) cell lines, and found that the expressions of HIF-1, VEGF, and IL- 8 significantly reduced in a dose-dependent manner. Additionally, cell invasion and migration were also found to have declined along with the suppressed expression of uPA [46]. In vitro and in vivo studies by Kanjoormana et al. revealed the anti-angiogenic characteristics of UA: UA inhibited capillary formation of the tumor induced by highly metastatic melanoma (B16F10) cells in C57BL/6 mice. Furthermore, UA-treated mice exhibited decreased levels of VEGF and proinflammatory cytokines in the serum compared to control mice. Likewise, UA-treated B16F-10 cells have decreased expression of VEGF and iNOS as well as an inhibition of MMP-2 and MMP-9, thereby supporting the in vivo results [47]. In another study, Jin et al. using nano-particle technology (UA loaded chitosan nanoparticle) for drug delivery and discovered a significant decrease in angiogenesis of chicken chorioallantoic membranes (CAM) in vivo for a dose 10 times lower than the normal drug delivery system [48]. Moreover, in an in vivo study of prostate cancer, Shanmugam et al. found that UA inhibits cell proliferation, invasion, and metastasis, as well as angiogenesis to prevent tumor growth through the suppression of NF- $\kappa B$ and STAT3 pathways [49]. Another study showed that UA is able to inhibit crucial steps of angiogenesis, as well as endothelial cell proliferation, migration, and differentiation in vitro and inhibition of $\mathrm{CAM}$ in vivo. However, it also induces other steps of angiogenesis, such as ECM degradation by MMP-2 and urokinase, indicating that a more comprehensive analysis of the role of UA in angiogenesis is required [50]. These results support the notion that UA has anti-angiogenic properties that inhibit carcinogenesis.

\section{Antioxidants}

Reactive oxygen species (ROS) are normally intracellularly produced by mitochondria and cleared by the cell's antioxidant defense mechanisms. An inability to clear ROS, and the subsequent excessive intracellular accumulation of ROS, can promote cancer. This explains why cancer cells are often characterized by excessive intracellular ROS. Transcription factors such as NF- $\mathrm{kB}$ and STAT3, kinases, growth factors, cytokines, and enzymes affect numerous signaling pathways that are further modulated by ROS, and these pathways are associated with cellular transformation, inflammation, tumorigenesis, proliferation, metastasis, invasion, and angiogenesis [51]. Some derivatives of UA when administered to NTUB1 cells (a human bladder cancer cell line) result in cell cycle arrest by increased levels of ROS in these cancer cells [52]. Ramos et al. found that Caco-2 cells pretreated with UA before being subjected to oxidative injury are protective against $\mathrm{H} 2 \mathrm{O} 2$ DNA damage and also increase DNA repair. Therefore, this property of UA can contribute to combating the carcinogenic effects arising from ROS [53]. Gayathri et al. by in vivo examination of rats with hepatocarcinogenesis induced by diethylnitrosamine, showed a reduced amount of oxidative stress following UA treatment, thereby suggesting a role for UA as a chemopreventive agent for diseases facilitated by free radicals [54]. Yang et al. recently demonstrated the anti-proliferative effect of UA in human adenocarcinoma gastric cancer (BGC-823) cells and found that UA could sensitize cells to radiotherapy; this enhanced, synergistic treatment approach is linked with elevated activity of ROS, G2/M cell cycle arrest, and apoptosis [55]. In addition, Lin et al. showed that UA in conjunction with cisplatin causes an increase in p21, cyclin E, and p53 levels as well as a decrease in p27 and cyclin D1 proteins. Moreover, p-p38 was elevated by the increase in ROS-induced cell death of bladder cancer (NTUB1) cells [56]. Another study confirmed that the ethanolic extract of Wrightia tomentosa, which contains UA and olenolic acid, exhibits anti-breast cancer activity via G1 cell cycle arrest, generation of ROS, loss of mitochondrial membrane potential, and apoptosis of MCF-7 and MDA-MB-231 cells in vitro. Gao et al. also found a similar effect using modified UA $[57,58]$.

\section{Anti-inflammatory}

Inflammation is an intricate occurrence linked to numerous diseases, including cancer. Anti-inflammatory activity of UA was observed with the reduced production of pro-inflammatory cytokines 
(e.g., IL-2, IFN- $\gamma$, and TNF- $\alpha$ ) by Th-1 cells after the use of UA in vivo [59]. UA was also found to inhibit tumor growth and accentuate the survival rate of mice by decreasing inflammation-inducing mediators (i.e., STAT3, AKT, and IKKa/b) [60]. In addition, when arthritic BALB/c mice were treated with UA, the production of pro-inflammatory cytokines (e.g., IL-2, IFN- $\gamma$, and TNF-) by Th-1 cells was reduced [59]. Takada et al. found that in human umbilical vein endothelial cells (HUVECs), UA causes a decrease in the expression of E-selectin via the inhibition of NF- $\kappa B$ translocation to the nucleus [61]. Santos et al. found that UA causes decrease in histamine release, inhibition of prostaglandin, and production of leukotrienes [62]. Prasad et al. investigated chemoresistant human pancreatic cancer and found that UA sensitizes pancreatic cancer cells to gemcitabine [63]. They used AsPC-1, MIA PaCa-2, and Panc-28 cells as well as nude mice implanted with Panc-28 cells for in vitro and in vivo studies to show the antiproliferative and proapoptotic properties of UA [63]. Moreover, UA has the ability to regulate proteins related to proliferation, metastasis, and angiogenesis by inactivating NF- $\mathrm{\kappa B}$ and STAT3 signaling pathways responsible for inflammation [63]. A study conducted by Suh et al. using mouse macrophage RAW264.7 cells found that the expressions of inducible nitric oxide (iNOS) and cyclooxygenase (COX-2) were diminished with the inhibition of NF- $\mathrm{KB}$ as a result of UA pretreatment [64]. Similarly, another study on human mammary epithelial cells showed that treatment of UA suppresses the amount of COX-2 protein induced by TPA (12-o-tetra decanoylphorbol-13-acetate) and inhibits the synthesis of PGE2 (prostaglandin E2) in 184B5/HER cells [65]. Furthermore, Kowalczyk et al. discovered that UA is potentially effective in preventing skin tumor formation and that it decreases inflammatory signaling by downregulating COX-2 and IL- 6 expression in a mouse model [66]. Another study on skin tumorigenesis by Huang et al. discovered that rosemary extract (which contains a substantial quantity of UA) when applied to the skin, inhibits TPA-induced inflammation and tumorigenesis in mice in a dose-dependent manner [67].

\section{Autophagy}

Autophagy is an evolutionarily conserved cellular process first observed in yeast and is characterized by the degradation of cellular content into by-products that are reused in other physiological processes for cell survival. However, excess autophagy is also linked to autophagic cell death. Autophagy is found to be closely associated with tumorigenesis, and excessive autophagic degradation of the cellular components in cancer cells can induce cell death, eventually preventing neoplasia [68-70]. Junco et al. revealed that UA synergizes with other therapeutic drugs to attenuate the viability of melanoma cells by inhibiting excessive autophagy. Paradoxically, the level of LC3II was found to be increased, indicating an accumulation of autophagosomes. However, UA inhibits the pro-autophagic proteins Beclin-1 and p62, which shows that UA decreases cell viability of melanoma (i.e., B16F10 and A375 cells) cells in combination with resveratrol and chloroquine [71]. Another investigation involving malignant glioma (U87MG) cells by Shen et al. revealed that UA triggers G1 phase cell cycle arrest and induces autophagy characterized by the generation of acidic vesicular organelles, an increase in the levels of autophagolysosomes and LC3II. In addition, UA causes ER-stress and an increase in intracellular calcium accompanied by ROS production, indicating that ER stress leads to the process of autophagy [72]. In a study of cervical cancer (TC-1) cells, cell death due to the induction of autophagy was observed instead of apoptosis, which is customarily triggered. Furthermore, the inhibition of autophagy by wortmannin and by silencing the ATG5 gene with siRNA reduced the expression of LC3II and increased the viability of UA-treated TC-1 cells [73]. Zhao et al. found that treatment of UA on breast cancer (MCF-7) cells induced both ER stress as well as autophagy. The ER stress caused by autophagy protected the cells from UA-induced apoptosis by the upregulation of MCL-1 mediated through EIF2AK3 (eukaryotic translation initiation factor 2- $\alpha$ kinase 3). Moreover, the activation of MAPK1/3 was the contributing factor for the protection of cells via UA-induced autophagy [74]. Similarly, a study in apoptosis-resistant colorectal cancer (HCT15) cells indicated that UA induces cell death of HCT15 by activating an apoptotic pathway and modulating autophagy through the c-jun-N-terminal kinase (JNK) pathway [75]. Furthermore, Shin et al. reported that UA induces apoptosis and autophagy, including a G1 phase cell cycle arrest in prostate cancer (PC3) cells. The inhibition of autophagy with the help of 3-methyladenine or Beclin-1/Atg5 small interfering RNA increases UA-induced apoptosis, suggesting that autophagy acts as a cell survival mechanism. Hence, the combination of an autophagy inhibitor and UA could be a potential novel approach for cancer therapy [76]. Therefore, UA-induced autophagy in conjunction with apoptosis-based therapies is an emerging strategy for cancer therapy $[77,78]$.

\section{Conclusion}

This review attempts to summarize the therapeutic potential of UA in carcinogenesis both in vivo and in vitro. Natural compounds, $\mathrm{UA}$, have been proven to be effective in treating cancer or preventing its development. UA can affect multiple molecular signaling pathways linked to human carcinogenesis, apoptosis, inflammation, oxidative stress, angiogenesis, metastasis, autophagy, and proliferation. UA's synergistic effect with other therapeutic medications, such as chemotherapy and radiotherapy, can be a very effective approach to cure cancer. UA has a great safety and efficacy profile, but due to its limited water solubility and low bioavailability, it has not yet been successfully employed in clinical practice. Therefore, further extensive laboratory investigation and clinical trials are required to identify novel cellular target proteins and pathways involved in the therapeutic action of UA.

\section{Acknowledgements}

This research was supported by the Basic Science Research Program provided by the National Research Foundation of Korea (NRF) grant funded by the Korean government (MISP) (No. 2008-0062279 and No. 2015R1C1A2A01054054).

\section{References}

1. Koehn FE, Carter GT (2005) The evolving role of natural products in drug discovery. Nat Rev Drug Discov 4: 206-220.

2. Shanmugam MK, Dai X, Kumar AP, Tan BK, Sethi G, et al. (2013) Ursolic acid in cancer prevention and treatment: molecular targets, pharmacokinetics and clinical studies. Biochem Pharmacol 85: 1579-1587.

3. Sheng H, Sun H (2011) Synthesis, biology and clinical significance of pentacyclic triterpenes: a multi-target approach to prevention and treatment of metabolic and vascular diseases. Nat Prod Rep 28: 543-593.

4. Siegel RL, Miller KD, Jemal A (2015) Cancer statistics, 2015. CA Cancer J Clin 2015 65: 5-29.

5. Ferlay J, Soerjomataram I, Dikshit R, Eser S, Mathers C, et al. (2015) Cancer incidence and mortality worldwide: sources, methods and major patterns in GLOBOCAN 2012. Int J Cancer 136: E359-86.

6. Dar BA, Lone AM, Shah WA, Qurishi MA (2016) Synthesis and screening of ursolic acid-benzylidine derivatives as potential anti-cancer agents. Eur J Med Chem 111: 26-32.

7. He X, Liu RH (2007) Triterpenoids isolated from apple peels have potent antiproliferative activity and may be partially responsible for apple's anticancer activity. J Agric Food Chem 55: 4366-1370.

8. Baek JH, Lee YS, Kang CM, Kim JA, Kwon KS, et al. (1997) Intracellular Ca2+ release mediates ursolic acid-induced apoptosis in human leukemic HL-60 cells. Int J Cancer 73: 725-728.

9. Kim ES, Moon A (2015) Ursolic acid inhibits the invasive phenotype of SNU-484 human gastric cancer cells. Oncol Lett 9: 897-902. 
Citation: Navin R, Kim SM (2016) Therapeutic Interventions Using Ursolic Acid for Cancer Treatment. Med chem (Los Angeles) 6: 339-344. doi:10.4172/2161-0444.1000367

10. Wozniak L, Skapska S, Marszalek K (2015) Ursolic Acid-A Pentacyclic Triterpenoid with a Wide Spectrum of Pharmacological Activities. Molecules 20: 20614-20641.

11. Lin J, Chen Y, Wei L, Hong Z, Sferra TJ, et al. (2013) Ursolic acid inhibits colorectal cancer angiogenesis through suppression of multiple signaling pathways. Int J Oncol 43: 1666-16674.

12. Bonaccorsi I, Altieri F, Sciamanna I, Oricchio E, Grillo C, et al. (2008) Endogenous reverse transcriptase as a mediator of ursolic acid's antiproliferative and differentiating effects in human cancer cell lines. Cancer Lett 263: 130-139.

13. Yin MC, Lin MC, Mong MC, Lin CY (2012) Bioavailability, distribution, and antioxidative effects of selected triterpenes in mice. J Agric Food Chem 60: 7697-7701.

14. Shanmugam MK, Nguyen AH, Kumar AP, Tan BK, Sethi G (2012) Targeted inhibition of tumor proliferation, survival, and metastasis by pentacyclic triterpenoids: potential role in prevention and therapy of cancer. Cancer Lett 320: $158-170$.

15. Tsai SJ, Yin MC (2008) Antioxidative and anti-inflammatory protection of oleanolic acid and ursolic acid in PC12 cells. J Food Sci 73: H174-178.

16. Qian Z, Wang X, Song Z, Zhang H, Zhou S, et al. (2015) A phase I trial to evaluate the multiple-dose safety and antitumor activity of ursolic acid liposomes in subjects with advanced solid tumors. Biomed Res Int 2015: 809714

17. Tuli HS, Sharma AK, Sandhu SS, Kashyap D (2013) Cordycepin: a bioactive metabolite with therapeutic potential. Life Sci 93: 863-869.

18. Kim DK, Baek JH, Kang CM, Yoo MA, Sung JW, et al. (2000) Apoptotic activity of ursolic acid may correlate with the inhibition of initiation of DNA replication. Int J Cancer 87: 629-636.

19. Lauthier F, Taillet L, Trouillas P, Delage C, Simon A (2000) Ursolic acid triggers calcium-dependent apoptosis in human Daudi cells. Anticancer Drugs 11: 737 745.

20. Shan JZ, Xuan YY, Zheng S, Dong Q, Zhang SZ (2009) Ursolic acid inhibits proliferation and induces apoptosis of HT-29 colon cancer cells by inhibiting the EGFR/MAPK pathway. J Zhejiang Univ Sci B 10: 668-674.

21. Weng H, Tan ZJ, Hu YP, Shu YJ, Bao RF, et al. (2014) Ursolic acid induces cell cycle arrest and apoptosis of gallbladder carcinoma cells. Cancer Cell Int 14: 96.

22. Zheng QY, Li PP, Jin FS, Yao C, Zhang GH, et al. (2013) Ursolic acid induces ER stress response to activate ASK1-JNK signaling and induce apoptosis in human bladder cancer T24 cells. Cell Signal 25: 206-213.

23. Kim KH, Seo HS, Choi HS, Choi I, Shin YC, et al. (2011) Induction of apoptotic cell death by ursolic acid through mitochondrial death pathway and extrinsic death receptor pathway in MDA-MB-231 cells. Arch Pharm Res 34: 1363-1372.

24. Wang JS, Ren TN, Xi T (2012) Ursolic acid induces apoptosis by suppressing the expression of FoxM1 in MCF-7 human breast cancer cells. Med Oncol 29 10-15.

25. Li Y, Lu X, Qi H, Li X, Xiao X, et al. (2014) Ursolic acid induces apoptosis through mitochondrial intrinsic pathway and suppression of ERK1/2 MAPK in HeLa cells. J Pharmacol Sci 125: 202-210.

26. Xavier CP, Lima CF, Preto A, Seruca R, Fernandes-Ferreira M, et al. (2009) Luteolin, quercetin and ursolic acid are potent inhibitors of proliferation and inducers of apoptosis in both KRAS and BRAF mutated human colorectal cancer cells. Cancer Lett 281: 162-170.

27. Nam H, Kim MM (2013) Ursolic acid induces apoptosis of SW480 cells via p53 activation. Food Chem Toxicol 62: 579-583.

28. Wang $X$, Zhang F, Yang L, Mei Y, Long H, et al. (2011) Ursolic acid inhibits proliferation and induces apoptosis of cancer cells in vitro and in vivo. J Biomed Biotechnol 2011: 419343

29. Wang J, Li Y, Wang X, Jiang C, et al. (2012) Ursolic acid inhibits proliferation and induces apoptosis in human glioblastoma cell lines $\mathrm{U} 251$ by suppressing TGF-beta1/miR-21/PDCD4 pathway. Basic Clin Pharmacol Toxicol 111: 106112.

30. Shyu MH, Kao TC, Yen GC (2010) Oleanolic acid and ursolic acid induce apoptosis in $\mathrm{HuH} 7$ human hepatocellular carcinoma cells through a mitochondrial-dependent pathway and downregulation of XIAP. J Agric Food Chem 58: 6110-6118.

31. Harmand PO, Duval R, Delage C, Simon A (2005) Ursolic acid induces apoptosis through mitochondrial intrinsic pathway and caspase- 3 activation in M4Beu melanoma cells. Int J Cancer 114: 1-11.

32. Manu KA, Kuttan G (2008) Ursolic acid induces apoptosis by activating p53 and caspase-3 gene expressions and suppressing NF-kappaB mediated activation of bcl-2 in B16F-10 melanoma cells. Int Immunopharmacol 8: 974-981.

33. Wu B, Wang X, Chi ZF, Hu R, Zhang R, et al. (2012) Ursolic acid-induced apoptosis in K562 cells involving upregulation of PTEN gene expression and inactivation of the PI3K/Akt pathway. Arch Pharm Res 35: 543-548.

34. Leal AS, Wang R, Salvador JA, Jing Y (2012) Synthesis of novel ursolic acid heterocyclic derivatives with improved abilities of antiproliferation and induction of p53, p21waf1 and NOXA in pancreatic cancer cells. Bioorg Med Chem 20: 5774-5786.

35. Li J, Guo WJ, Yang QY (2002) Effects of ursolic acid and oleanolic acid on human colon carcinoma cell line HCT15. World J Gastroenterol 8: 493-495.

36. Kumar G, Tuli HS, Mittal S, Shandilya JK, Tiwari A, et al. (2015) Isothiocyanates: a class of bioactive metabolites with chemopreventive potential. Tumour Biol 36: 4005-4016.

37. Xiang L, Chi T, Tang Q, Yang X, Ou M, et al. (2015) A pentacyclic triterpene natural product, ursolic acid and its prodrug US597 inhibit targets within cel adhesion pathway and prevent cancer metastasis. Oncotarget 6: 9295-9312.

38. Jedinák A, Mucková M, Kost'álová D, Maliar T, Masterova I (2006) Antiprotease and antimetastatic activity of ursolic acid isolated from Salvia officinalis. Z Naturforsch C 61: 777-782.

39. Huang HC, Huang CY, Lin-Shiau SY, Lin JK (2009) Ursolic acid inhibits IL1 beta or TNF-alpha-induced C6 glioma invasion through suppressing the association ZIP/p62 with PKC-zeta and downregulating the MMP-9 expression. Mol Carcinog 48: 517-531.

40. Liu K, Guo L, Miao L, Bao W, Yang J, et al. (2013) Ursolic acid inhibits epithelialmesenchymal transition by suppressing the expression of astrocyte-elevated gene-1 in human nonsmall cell lung cancer A549 cells. Anticancer Drugs 24: 494-503.

41. Prasad S, Yadav VR, Sung B, Reuter S, Kannappan R, et al. (2012) Ursolic acid inhibits growth and metastasis of human colorectal cancer in an orthotopic nude mouse model by targeting multiple cell signaling pathways: chemosensitization with capecitabine. Clin Cancer Res 18: 4942-4953.

42. Yeh CT, Wu CH, Yen GC (2010) Ursolic acid, a naturally occurring triterpenoid suppresses migration and invasion of human breast cancer cells by modulating c-Jun N-terminal kinase, Akt and mammalian target of rapamycin signaling. Mol Nutr Food Res 54: 1285-1295.

43. Cha HJ, Bae SK, Lee HY, Lee OH, Sato H, et al. (1996) Anti-invasive activity of ursolic acid correlates with the reduced expression of matrix metalloproteinase- 9 (MMP-9) in HT1080 human fibrosarcoma cells. Cancer Res 56: 2281-2284.

44. Shanmugam MK, Manu KA, Ong TH, Ramachandran L, Surana R, et al. (2011) Inhibition of CXCR4/CXCL12 signaling axis by ursolic acid leads to suppression of metastasis in transgenic adenocarcinoma of mouse prostate model. Int $J$ Cancer 129: 1552-1563.

45. Saraswati S, Agrawal SS, Alhaider AA (2013) Ursolic acid inhibits tumor angiogenesis and induces apoptosis through mitochondrial-dependent pathway in Ehrlich ascites carcinoma tumor. Chem Biol Interact 206: 153-165.

46. Lin CC, Huang CY, Mong MC, Chan CY, Yin MC (2011) Antiangiogenic potential of three triterpenic acids in human liver cancer cells. J Agric Food Chem 59: 755-762.

47. Kanjoormana M, Kuttan G (2010) Antiangiogenic activity of ursolic acid. Integr Cancer Ther 9: 224-235.

48. Jin H, Pi J, Yang F, Wu C, Cheng X, et al. (2016) Ursolic acid-loaded chitosan nanoparticles induce potent anti-angiogenesis in tumor. Appl Microbiol Biotechnol.

49. Shanmugam MK, Rajendran P, Li F, Nema T, Vali S, et al. (2011) Ursolic acid inhibits multiple cell survival pathways leading to suppression of growth of prostate cancer xenograft in nude mice. J Mol Med (Berl) 89: 713-727.

50. Cárdenas C, Quesada AR, Medina MA (2004) Effects of ursolic acid on different steps of the angiogenic process. Biochem Biophys Res Commun 320: 402-408.

51. Prasad S, Gupta SC, Tyagi AK (2016) Reactive oxygen species (ROS) and cancer: Role of antioxidative nutraceuticals. Cancer Lett.

52. Tu HY, Huang AM, Wei BL, Gan KH, Hour TC, et al. (2009) Ursolic acid derivatives induce cell cycle arrest and apoptosis in NTUB1 cells associated with reactive oxygen species. Bioorg Med Chem 17: 7265-7274. 
Citation: Navin R, Kim SM (2016) Therapeutic Interventions Using Ursolic Acid for Cancer Treatment. Med chem (Los Angeles) 6: 339-344. doi:10.4172/2161-0444.1000367

53. Ramos AA, Pereira-Wilson C, Collins AR (2010) Protective effects of ursolic acid and luteolin against oxidative DNA damage include enhancement of DNA repair in Caco-2 cells. Mutat Res 692: 6-11.

54. Gayathri R, Priya DK, Gunassekaran GR, Sakthisekaran D (2009) Ursolic acid attenuates oxidative stress-mediated hepatocellular carcinoma induction by diethylnitrosamine in male Wistar rats. Asian Pac $\mathrm{J}$ Cancer Prev 10: 933-938.

55. Yang Y, Jiang M, Hu J, Lv X, Yu L, et al. (2015) Enhancement of Radiation Effects by Ursolic Acid in BGC-823 Human Adenocarcinoma Gastric Cancer Cell Line. PLoS One 10: e0133169.

56. Lin KW, Huang AM, Lin CC, Chang CC, Hsu WC, et al. (2014) Anti-cancer effects of ursane triterpenoid as a single agent and in combination with cisplatin in bladder cancer. Eur J Pharmacol 740: 742-751.

57. Chakravarti B, Maurya R, Siddiqui JA, Bid HK, Rajendran SM, et al. (2012) In vitro anti-breast cancer activity of ethanolic extract of Wrightia tomentosa: role of pro-apoptotic effects of oleanolic acid and urosolic acid. J Ethnopharmacol 142: $72-79$.

58. Gao J, Li X, Gu G, Liu S, Cui M, et al. (2012) Facile synthesis of triterpenoid saponins bearing $\beta$-Glu/Gal- $(1 \rightarrow 3)-\beta$-GluA methyl ester and their cytotoxic activities. Bioorg Med Chem Lett 22: 2396-2400.

59. Ahmad SF, Khan B, Bani S, Suri KA, Satti NK, et al. (2006) Amelioration of adjuvant-induced arthritis by ursolic acid through altered Th1/Th2 cytokine production. Pharmacol Res 53: 233-240.

60. Ma JQ, Ding J, Xiao ZH, Liu CM (2014) Ursolic acid ameliorates carbon tetrachloride-induced oxidative DNA damage and inflammation in mouse kidney by inhibiting the STAT3 and NF-î́B activities. Int Immunopharmacol 21: 389-395.

61. Takada K, Nakane T, Masuda K, Ishii H (2010) Ursolic acid and oleanolic acid, members of pentacyclic triterpenoid acids, suppress TNF-alphainduced E-selectin expression by cultured umbilical vein endothelial cells. Phytomedicine 17: 1114-1119.

62. Santos Rosa C, García Gimenez MD, Saenz Rodriguez MT, De la Puerta Vazquez R (2007) Antihistaminic and antieicosanoid effects of oleanolic and ursolic acid fraction from Helichrysum picardii. Pharmazie 62: 459-462.

63. Prasad S, Yadav VR, Sung B, Gupta SC, Tyagi AK, et al. (2016) Ursolic acid inhibits the growth of human pancreatic cancer and enhances the antitumor potential of gemcitabine in an orthotopic mouse model through suppression of the inflammatory microenvironment. Oncotarget 7537.

64. Suh N, Honda T, Finlay HJ, Barchowsky A, Williams C, et al. (1998) Novel triterpenoids suppress inducible nitric oxide synthase (iNOS) and inducible cyclooxygenase (COX-2) in mouse macrophages. Cancer Res 58: 717-723.

65. Subbaramaiah K, Michaluart P, Sporn MB, Dannenberg AJ (2000) Ursolic acid inhibits cyclooxygenase-2 transcription in human mammary epithelial cells. Cancer Res 60: 2399-2404.

66. Kowalczyk MC, Junco JJ, Kowalczyk P, Tolstykh O, Hanausek M, et al. (2013) Effects of combined phytochemicals on skin tumorigenesis in SENCAR mice. Int J Oncol 43: 911-918.

67. Huang MT, Ho CT, Wang ZY, Ferraro T, Lou YR, et al. (1994) Inhibition of skin tumorigenesis by rosemary and its constituents carnosol and ursolic acid. Cancer Res 54: 701-708.

68. Mathew R, Kongara S, Beaudoin B, Karp CM, Bray K, et al. (2007) Autophagy suppresses tumor progression by limiting chromosomal instability. Genes Dev 21: $1367-1381$.

69. McPhee CK, Logan MA, Freeman MR, Baehrecke EH (2010) Activation of autophagy during cell death requires the engulfment receptor Draper. Nature 465: 1093-1096.

70. Li P, Du Q, Cao Z, Guo Z, Evankovich J, et al. (2012) Interferon-gamma induces autophagy with growth inhibition and cell death in human hepatocellular carcinoma (HCC) cells through interferon-regulatory factor-1 (IRF-1). Cancer Lett 314: 213-222.

71. Junco JJ, Mancha-Ramirez A, Malik G, Wei SJ, Kim DJ, et al. (2015) Ursolic acid and resveratrol synergize with chloroquine to reduce melanoma cell viability. Melanoma Res 25: 103-112.

72. Shen S, Zhang Y, Zhang R, Tu X, Gong X (2014) Ursolic acid induces autophagy in U87MG cells via ROS-dependent endoplasmic reticulum stress. Chem Biol Interact 218: 28-41.

73. Leng S, Hao Y, Du D, Xie S, Hong L, et al. (2013) Ursolic acid promotes cancer cell death by inducing Atg5-dependent autophagy. Int J Cancer 133: 27812790.

74. Zhao C, Yin S, Dong Y, Guo X, Fan L, et al. (2013) Autophagy-dependent EIF2AK3 activation compromises ursolic acid-induced apoptosis through upregulation of MCL1 in MCF-7 human breast cancer cells. Autophagy 9: 196207.

75. Xavier CP, Lima CF, Pedro DF, Wilson JM, Kristiansen K, et al. (2013) Ursolic acid induces cell death and modulates autophagy through JNK pathway in apoptosis-resistant colorectal cancer cells. J Nutr Biochem 24: 706-712.

76. Shin SW, Kim SY, Park JW (2012) Autophagy inhibition enhances ursolic acidinduced apoptosis in PC3 cells. Biochim Biophys Acta 1823: 451-457.

77. Chi S, Kitanaka C, Noguchi K, Mochizuki T, Nagashima Y, et al. (1999) Oncogenic Ras triggers cell suicide through the activation of a caspaseindependent cell death program in human cancer cells. Oncogene 18: 2281 2290.

78. Zhou F, Yang Y, Xing D (2011) Bcl-2 and Bcl-xL play important roles in the crosstalk between autophagy and apoptosis. FEBS J 278: 403-413. 